\title{
Dancing effects on the magnitude of the vestibular-cervical reflex
}

\author{
Oliveira-Barreto AC ${ }^{* 1}$, Menezes PL ${ }^{1}$, Feitosa ABL ${ }^{1}$, Oliveira PF ${ }^{1}$, Taguchi CK ${ }^{1}$, Passos PS ${ }^{1}$ and Pereira LD ${ }^{1}$ \\ ${ }^{1}$ Universidade Federal de São Paulo, Brazil
}

\begin{abstract}
Objective: Determine the influence of dance movements on the vestibular system and compare the response magnitude of the vestibulospinal pathway between dancers and non-dancers.

Methodology: The sample consisted of 61 subjects, aged between 18 and 35 years, 31 dancers and 30 controls. Cervical VEMP recordings were taken in the sternocleidomastoid muscle. A total of 100 tone-burst stimuli were promediated at a frequency of $500 \mathrm{~Hz}$, intensity of $95 \mathrm{~dB}$ NAn. Results: The cVEMP of the dancers exhibited waves earlier and with greater amplitudes than those of the controls. A significant intergroup difference was observed for n 23 latency and n 23 amplitude and p13-n23 interlatency.
\end{abstract}

Conclusion: Greater amplitudes and lower vestibulospinal reflex latencies were observed for dancers compared to non-dancers.

\section{Introduction}

Dance, specifically classical ballet, involves exercises that produce efficient muscle power and motor coordination. Integration is needed between the osteomuscular and central nervous systems (SNC), with kinesthetic feedback acting as an adjustment element [1].

Dance promotes head and trunk movements, and displacement from the center of gravity in all directions and from the support axis enables the development of factors that contribute to maintaining balance, such as joint mobility and coordination [2] [Swathi and Kumar, 2013]. A number of reports suggest that dance training improves balance in young people and adults $[3,4]$.

During dance, the need for constant positioning of the head in different directions in relation to gravity activates the vestibulospinal reflex, which causes compensatory displacement of the cephalic segment in the direction opposite to body rotation, keeping the head stable $[1,5]$.

Given that the vestibulospinal reflex is important for postural stabilization, knowledge regarding the effects of dance on the magnitude of this reflex may contribute not only to basic neuroscience, but could also strongly affect neurorehabilitation techniques $[5,6]$. No significant studies were found that assess this reflex in classical ballet dancers, only two with small samples involving different dance styles (Egyptian dance or salsa) [2,7].

Thus, the aim was to determine the influence of dance movements on the vestibular system and compare the response magnitude of the vestibulospinal pathway between dancers and non-dancers.

\section{Methods}

This study was conducted according to the principles expressed in the Declaration of Helsinki and complies with resolution 466/2012 of the National Health Council, for studies with human beings, and was approved by the University of São Paulo Research Ethics Committee, under protocol no. 20907713.5.0000.5505. This is a cross-sectional, analytical observational study conducted at the Audiology outpatient facility of a public university.

The sample consisted of 61 age and sex-matched individuals, $31 \mathrm{c}$ (test group) and 30 non-dancers (control group), aged between 18 and 35 years, with normal auditory sensitivity. The group of dancers had to have at least three years of professional ballet experience and the control group self-reported not being professional musicians or dancers. The subjects could have no history of auditory and/or vestibular alterations, exposure to occupational or leisure noise, or earwax or foreign body in the external acoustic meatus.

After the individuals screened to take part in the study gave their informed consent, they were submitted to the cervical vestibular evoked myogenic potential (cVEMP) test using an Interacoustics Eclipse EP-25 audiometer.

This potential is captured by surface electrodes placed on the skin and fixed with adhesive tape, after cleaning with abrasive paste, alcohol, cotton and prior use of electrolyte paste. The active electrode was placed on the upper half of the sternocleidomastoid muscle, ipsilateral to the stimulation, the reference electrode on the upper edge of the ipsilateral sternum, and the ground electrode on the frontal midline. Impedance was measured between the electrodes, allowing a difference of up to 3 $\mathrm{k} \Omega$, and of each electrode, allowing a difference of $5 \mathrm{k} \Omega$.

To obtain the cVEMP recording in the sternocleidomastoid muscle, the patient remained seated, with maximum lateral head rotation to the

Correspondence to: Aline Cabral de Oliveira-Barreto, Inácio Barbosa Av., 1100, Aruana, Aracaju-SE, Brazil; Tel: +55 79 999211700. E-mail: alinecabralbarreto@ gmail.com

Key words: Epostur, postural balance, vestibular evoked myogenic potentials, motion perception

Received: March 11, 2017; Accepted: April 10, 2017; Published: April 13, 2017 
side contralateral to the stimulus, maintaining tonic muscle contraction of between 60 and $80 \mu \mathrm{V}$, controlled by surface electromyography. The stimuli, presented through ER-3A insert earphones, were initiated in the afferent right, and then repeated in the afferent left.

In the cVEMP test, 100 tone-burst stimuli were promediated at a frequency of $500 \mathrm{~Hz}$, with a duration of 10 milliseconds (rise: $4 \mathrm{~ms}$, plateau: $2 \mathrm{~ms}$, fall: $4 \mathrm{~ms}$ ), repetition rate of $5 \mathrm{~Hz}$, and sound intensity level of $95 \mathrm{~dB}$ NAn, using a bandpass filter between 5 and $2200 \mathrm{~Hz}$. The recordings were made in $50 \mathrm{~ms}$ windows.

P13 latency was defined as the negative polarity of a biphasic wave that appears in approximately $13 \mathrm{~ms}$, and $\mathrm{n} 23$ latency as the positive polarity of the biphasic wave that appears in around $23 \mathrm{~ms}$. The amplitude of the p13 wave was assessed from zero (reference) to the most negative peak of the wave, on the $\mathrm{Y}$ axis. The amplitude of the $\mathrm{n} 23$ wave was assessed from zero (reference) to the most positive peak of the wave, also on the $\mathrm{Y}$ axis. Both were observed in microvolts.

The data were tabulated and processed by SPSS 21.0 software. The Shapiro-Wilk test was applied to determine sample normality, and the student's T-Test or Mann-Whitney Test to make a comparative analysis between the two independent variables, depending on whether the sample exhibited normal or non-normal distribution, respectively. Alpha values $<0.05$ were considered significant. The beta value was 0.1 .

\section{Results}

The sample consisted of 61 subjects, namely 31 dancers $(15$ men $=$ $48.4 \%$ ), of which 16 were women (51.6\%), and 30 controls, 15 of each sex. The student's t-test showed no age difference between the dancers (22.48 \pm 4.8 years) and non-dancers ( $22.5 \pm 3.20$ years $)$, with a p-value of 0.97 .

There was no significant intergroup difference between the right and left ears, considering p-values $<0.05$ ), for the Student's T-test. In comparative analysis between the sexes, the cVEMP recorded in male dancers exhibited earlier (lower latencies) and more robust (greater amplitudes) p13 and n 23 waves than those captured in their female counterparts, but with no significantly different mean values between the sexes with $\mathrm{p}>0.05$. The same cVEMP analysis between the sexes in the control group obtained similar results between men and women, with no significant differences $(\mathrm{p}<0.05)$.

The cVEMP test showed earlier waves with greater amplitudes in the dancer group compared to non- dancer group (Figure 1). The Mann-Whitney test revealed a significant intergroup difference for $\mathrm{n} 23$ latency and amplitude and p13-n23 interlatency $(\mathrm{p}<0.05)$ (Table 1).

\section{Discussion}

The findings of this study showed no inter-sex difference for the dancer group and non-dancer group, in line with other investigations $[8,9]$. We observed that the n23 wave of cVEMP exhibited a higher amplitude and lower latency in the dancers than in non-dancers, similar to the findings of [2], but with significant differences for all p13 and 23 latency and amplitude parameters. These results may be due to the fact that dance requires more balance and therefore a more reactive vestibular system in dancers than in non-dancers [2].

For many years individuals have enjoyed the health benefits of dancing through its body movement techniques. This corporal perception during movement results in better body control in terms of posture, balance, laterality, among others [10]. During dance exercises, the body requires greater balance, increasing the neural discharge into

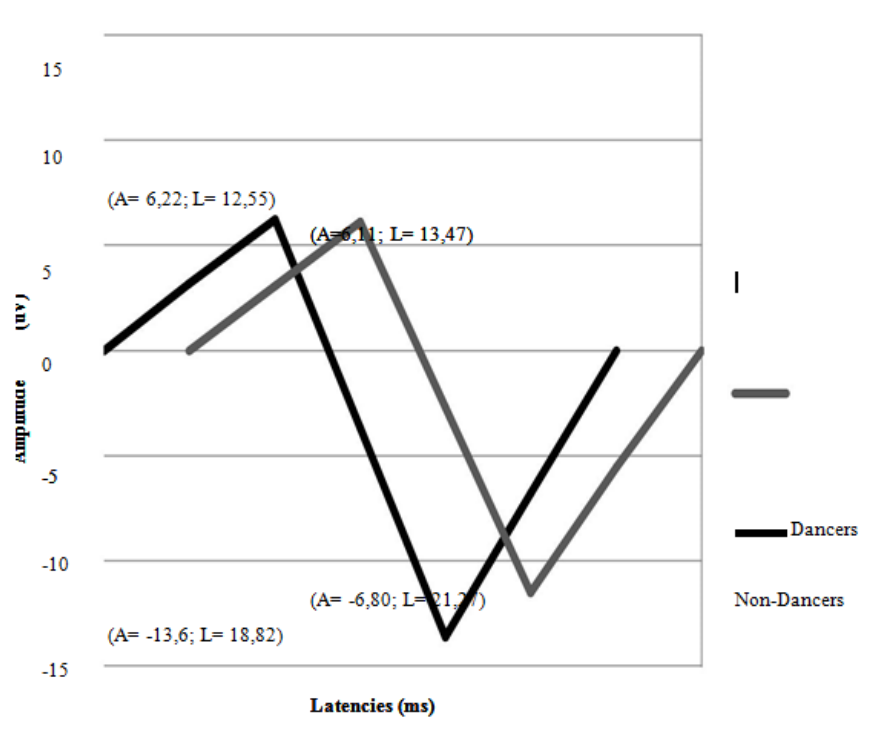

Figure 1. Mean Values of the Vestibular evoked potentials for dancers and non-dancers

Table 1. P-Values on the comparison of latency and amplitude of Cervical VEMP waves between dancers and non-dancers

\begin{tabular}{|c|c|c|c|c|}
\hline cVEMP Waves & Variables & Dancers & Non-Dancers & P-Value \\
\hline & Latency (ms) & $12,55 \pm 3,81$ & $13,47 \pm 3,59$ & $0,306^{* *}$ \\
\hline \multicolumn{5}{|l|}{ P13 } \\
\hline & Amplitude $(\mu \mathrm{V})$ & $6,22 \pm 5,81$ & $6,11 \pm 5,88$ & $0,678 * *$ \\
\hline & Latency (ms) & $18,82 \pm 3,84$ & $21,27 \pm 4,76$ & $0,005^{* \mathrm{~A}}$ \\
\hline \multicolumn{5}{|l|}{$\mathrm{N} 23$} \\
\hline & Amplitude $(\mu \mathrm{V})$ & $13,61 \pm 8,62$ & $11,53 \pm 10,30$ & 0,010 *A \\
\hline \multirow[t]{2}{*}{ P13-N23 } & Latency (ms) & $6,54 \pm 3,81$ & $7,24 \pm 4,53$ & $0,045^{* \mathrm{~A}}$ \\
\hline & Amplitude $(\mu \mathrm{V})$ & $7,81 \pm 5,24$ & $7,31 \pm 5,12$ & $0,212 *$ \\
\hline
\end{tabular}

*Test, **T Student Test; $\mu \mathrm{V}$ :

the vestibular system, in order to balance the body, which becomes more agile and in better equilibrium [2]. As a consequence of the movements executed during dance, the vestibular system can produce plasticity and the reactions in the vestibular organs of dancers may be different from those of non-dancers [7].

In contrast to our findings, a study conducted by [7] with 16 subjects, 8 dancers (Egyptian dance) and 8 non-dancers, showed no intergroup difference in latency or amplitude in cVEMP or Ocular VEMP (oVEMP). These results may be due to the fact that the study considered different dance styles.

The dance style selected here was classical ballet, considered a physical activity that requires musculoskeletal conditioning, due to the complex high-impact movements and large joint range of motion involved, in addition to developing coordination, balance and laterality associated with strength and flexibility of technical execution. Thus, developing these skills, associated with the movements inherent to ballet, may result in improved vestibulospinal reflex development [11].

\section{Conclusion}

Greater amplitudes and lower latencies were observed for the vestibulospinal reflex in dancers than in non-dancers.

\section{References}

1. Nastase VD (2012) The roll of sensations, perceptions and representations in learning dance sport. Procedia Soc Behav Sci 51: 957-960.

2. Swathi VM, Kumar SKN (2013) Influence of dance training on sacculo collic pathway: vestibular evoked myogenic potentials (vemp) as objective tool. $J$ of Evolution of Medical and Dental Sciences. 2: 7747-7754. 
3. Fedrici A, Bellagamba S, Rocchi MB (2005) Does dance-based training improve balance in adult and Young old subjects? A pilot randomized controlled trial. Aging Clin Exp Res. 17: 385-389. [Crossref]

4. Krampe J, Rantz MJ, Dowell L, Schamp R, Skubic M, et al. (2010) Dance-based therapy in a program of all-inclusive care for the elderly: an integrative approach to decrease fall risk. Nurs Adm Q 34: 156-161. [Crossref]

5. Kiefer AW, Riley MA, Shockley K, Sitton CA, Hewett TE, et al. (2011) Multisegmental postural coordination in professional ballet dancers. Gait \& Posture 34: 7680. [Crossref]

6. Pantev C, Herholz SC (2011) Plasticity of the human auditory cortex related to musical training. Neurosci Biobehav Rev 35: 2140-2154. [Crossref]
7. Sinha SK, Bohra V, Sanju HK (2013) Comparison of Cervical and Ocular Vestibular Evoked Myogenic Potentials in Dancers and Non-Dancers. Audiol Res 3: e6. [Crossref]

8. Carnaúba AT, Farias VV, Santos N, Oliveira AC, Rodrigues RG, et al. (2011) Influence of gender on the vestibular evoked myogenic potential. Braz J Otorhinolaryngol 77: 245-248. [Crossref]

9. Guillén VP, García EG, Piñero AG, Rey APD, Pérez CM (2005) Potencial vestibular miogénico evocado: un aporte al conocimiento de lafisiología y patología vestibular. Patrones cuantitavos em La población normal. Acta Otorrinolaringol Esp. 56: 349-353.

10. Fonseca CC, Vecchi RL, Gama EF (2012) A influência da dança de salão na percepção corporal. Motriz.

11. Thiesen T, Sumiya A (2011) Equilíbrio e arco plantar no balé clássico. Conscientia e Saúde 10: 138-142.

Copyright: (C2017 Oliveira-Barreto AC. This is an open-access article distributed under the terms of the Creative Commons Attribution License, which permits unrestricted use, distribution, and reproduction in any medium, provided the original author and source are credited. 